\title{
EFECTO DEL BENEFICIADO DE MEZCLAS DE VARIEDADES DE ARROZ SOBRE VARIABLES DE RENDIMIENTO MOLINERO ${ }^{1}$
}

\author{
Manuel Zeledón-Grau², Ronny Barboza-Mora², Minor Cruz-Varela ${ }^{3}$
}

\section{RESUMEN}

Efecto del beneficiado de mezclas de variedades de arroz sobre variables de rendimiento molinero. Con el fin de determinar si las variables de rendimiento molinero del arroz (rendimiento de molino, rendimiento de arroz comercial e índice de pilada) se afectan cuando una variedad se beneficia en mezcla con otras, en el Centro de Investigaciones en Granos y Semillas (CIGRAS) se evaluaron cuatro mezclas de las tres variedades más utilizadas en Costa Rica en la cosecha 1999-2000 (CR-1113, CR-5272 y Setesa-9). Otras variables analizadas fueron los porcentajes de: grano quebrado, grano quebrado grueso, puntilla, semolina (salvado de arroz), semolina pura y cascarilla en la semolina. Para cada variable se calculó un índice $\left(\mathrm{I}_{\mathrm{m}}\right)$ que expresa qué tanto el valor obtenido se desvió del valor esperado para cada una de las mezclas (esperado $=$ promedio de los resultados indivi duales para cada variedad cuando se la procesó sin mezclar). No se encontraron diferencias significativas entre los índices de los tratamientos evaluados. Sin embargo, se observó un efecto detrimental en las variables de rendimiento molinero de las mezclas en que participó CR 5272. Contrario a esto, la mezcla CR-1113-Setesa-9 mostró un efecto beneficioso. Los efectos detrimentales observados con el beneficiado de las mezclas fueron atribuidos a una mayor producción que la esperada de puntilla y de pedazos muy pequeños de arroz en la semolina. Por su parte, en la mezcla en la que se registró un efecto beneficioso hubo una menor producción que la esperada de semolina pura y de quebrado grueso. La pérdida económica que causaría la mezcla más perjudicial fue estimada en \$ 1 por tonelada métrica.

Palabras clave: Costa Rica, rendimiento de molienda, índice de pilado, porcentaje de grano quebrado, arroz.

\begin{abstract}
Effect of milling mixed batches of rice varieties on yield variables. During harvest, drying and storage operations, rice varieties (cultivars) are frequently mixed together. In order to establish the effect of milling mixed batches of rice on yield variables, an experiment was conducted in the Grains and Seeds Research Center with certified seed of the three most important cultivars produced in Costa Rica during the 1999-2000 harvest. Samples of $1 \mathrm{~kg}$ were prepared for each of the cultivars or half of two or a third of the three, and milled in a McGill No. 3 laboratory mill; actual yields were compared to the expected ones (expected yield $=$ average of the yields of each cultivar in the mix). Among milling variables studied were milling yield, head rice yield, and percentages of broken kernels, large broken kernels, bran, pure bran and related material in bran. An index was calculated for each variable tested $\left(I_{m}\right)$ to measure the deviation between the actual yield of a given cultivar mix and the expected one. No statistically significant effects were detected for the treatments evaluated. However, decreases in milling yield variables were consistently observed when the CR-5272 was present in the mix, regardless of the proportion. On the contrary, the mix of the other two cultivars (CR-1113, Setesa-9) showed improved milling yields. Reductions in milling yields were associated with larger productions of small broken kernels than expected, as well as with a greater presence of very small pieces of broken kernels in the bran. Improved milling yields were associated with smaller than expected percentages of bran and larger broken kernels. The most detrimental cultivar mix among those evaluated would cause an approximate loss of 1 dollar per metric ton of paddy rice processed.
\end{abstract}

Key words: Costa Rica, milling yield, head rice yield, percentage of broken kernels, rice.

\footnotetext{
1 Recibido: 21 de febrero, 2006. Aceptado: 2 de marzo, 2007. Este trabajo contiene resultados de la tesis de Ing. Agr. presentada por el tercer autor a la Escuela de Agronomía de la Universidad de Costa Rica.

2 Centro de Investigaciones en Granos y Semillas, Facultad de Ciencias Agroalimentarias, Universidad de Costa Rica, San José, Costa Rica. Correo electrónico: cigras@cariari.ucr.ac.cr; mzeledon@cariari.ucr.ac.cr; rbarboza@cariari.ucr.ac.cr

3 Corporación Arrocera, San José, Costa Rica. Correo electrónico: mcruz@conarroz.com
} 


\section{INTRODUCCIÓN}

En Costa Rica, al igual que en otros países, en los molinos arroceros se da con frecuencia la mezcla de lotes de diferentes variedades. Esto puede ocurrir antes, durante o después del secado. De tal forma que el producto que se beneficia es a menudo una mezcla de variedades en proporciones desconocidas. La mezcla de estos materiales puede darse intencionalmente, cuando se decide mezclar arroz de dos o tres silos antes del beneficio, con el propósito de mejorar alguna característica en el producto final.

El beneficio o molienda del arroz tiene entre sus etapas finales el blanqueado y el pulido. Estas consisten en remover total o parcialmente las capas celulares más externas; lo que da origen al arroz pilado, por un lado y a la semolina, por el otro, como principal subproducto. Al blanquear y pulir el grano de arroz se tiene, como objetivo universal, tratar de quebrarlo lo menos posible.

Blanqueado y pulido son dos procesos diferentes; sin embargo, en Costa Rica el verbo pulir o el sustantivo pulido se utilizan para hacer referencia a ambos simultáneamente. En adelante, en este trabajo, se utilizará esta última acepción. Un inadecuado proceso de pulido puede causar dos efectos negativos en el grano: el sobrepulido, que produce disminuciones de rendimiento e incremento en el contenido de granos quebrados; y la remoción incompleta de las capas externas, que los consumidores podrían objetar por el color del grano, o bien, rechazarlo si desarrollara olor a rancio.

Una vez beneficiado, el valor del arroz depende principalmente de la cantidad de grano entero. Castillo y Gaviria (2000) indican que cuando la relación de precios entre el grano entero y el grano quebrado es superior a dos, es importante hacer esfuerzos para disminuir los porcentajes de grano quebrado. En Costa Rica, el precio del grano quebrado es arbitrariamente fijado como la mitad de lo que vale el grano entero.

Aún sin considerar la relación anterior, el identificar los factores que aumentan o disminuyen la producción de grano quebrado, ha sido considerado importante en Costa Rica por haber probado ser un camino que lleva a formas más eficientes de producción.
Los factores que afectan el rendimiento del arroz como grano entero en el proceso de industrialización son varios. Sun y Siebenmorgen (1993) indican que uno de estos factores es la diferencia en el espesor y el grosor de los granos. Estos autores encontraron que la producción de grano entero se incrementó con un aumento en el grosor de los granos, hasta alcanzar un máximo y luego decreció. Por lo tanto, el quebrado fue mayor en las fracciones más delgadas.

Otro de estos factores lo indica Andrews (1992), citado por Sun y Siebenmorgen (1993), a saber, que cuando el tiempo de pulido se incrementa, la producción de grano quebrado crece y el grado de molienda aumenta. Zeledón y Corrales (1997), quienes confirmaron lo anterior, obtuvieron aumentos promedio de 2,2 puntos porcentuales en el contenido de grano quebrado, entre el menor y el mayor tiempo de pulido (5 y $40 \mathrm{seg}$ ).

Arrieta (1994) informó de diferencias en la producción de semolina de las variedades CR 1113, CR-5272 y CR-201, siendo la proporción de semolina pura (sin fragmentos de cascarilla o de arroz) en CR-5272 significativamente menor que en las otras. Además, indicó que CR-5272 tiene cerca de cuatro puntos porcentuales más de cariópside que las otras variedades; lo cual, unido al hecho de que produce menos semolina pura y menos quebrado muy fino (arroz quebrado en la semolina) que CR-201, CR-1113 y CR-1821, explica que el rendimiento de molino de CR-5272 fuera superior al de las otras variedades.

Por la menor producción de semolina, se podría pensar que CR-5272 es un material de rápido pulido. De ser esto cierto, la variedad CR-5272 podría entonces estar siendo sobrepulida cuando es procesada en mezcla con otras variedades, con consecuencias hasta ahora desconocidas. No se sabe si con el beneficiado de la mezcla de dos o más variedades de arroz se produce mayor porcentaje de arroz quebrado que el promedio que se obtendría si se procesaran por separado; tampoco se sabe el efecto sobre el rendimiento de molino, la producción de semolina y la producción de puntilla, así como sobre otras variables de importancia económica.

Determinar si dicho efecto se da o no y su magnitud es importante para esta industria, pues de encontrarse que una o más variables de rendimiento molinero 
son afectadas (en forma detrimental o beneficiosa) permitiría a los molinos tomar decisiones y poder valorar la viabilidad de hacer los ajustes necesarios en los procesos de recibo, secado, almacenamiento, beneficio y otros, para evitar mezclas indeseables o bien promover las beneficiosas.

Con el objetivo de determinar la existencia y la dimensión de estos efectos, se diseñó este experimento, en el que se utilizaron tres variedades importantes en Costa Rica en la cosecha 1999-2000.

\section{MATERIALES Y MÉTODOS}

El estudio se llevó a cabo en el Laboratorio de análisis de calidad de granos del Centro de Investigaciones en Granos y Semillas (CIGRAS) de la Facultad de Ciencias Agroalimentarias de la Universidad de Costa Rica, ubicado en San Pedro de Montes de Oca, durante el 2001 y el 2002. Un primer informe de los resultados fue presentado por Cruz (2002).

Se utilizaron las tres variedades de arroz de tipo largo con mayor área sembrada en la primera cosecha del período 1999/2000; a saber, CR-1113, CR-5272 y Setesa 9 (OFIARROZ 2000).

Para asegurar la identidad genética de los lotes, se utilizó semilla certificada procesada en el 2001. Ensayos preliminares indicaron que esos lotes tenían las características típicas de un arroz en cáscara de buena calidad listo para ser beneficiado (menos de $20 \%$ de grano quebrado y menos de $12 \%$ de contenido de humedad).

Unidad experimental. Un kilogramo de arroz en cáscara, de cada una de las tres variedades o de sus respectivas mezclas.

Tratamientos. Consistieron en mezclas homogeneizadas de las variedades, según el detalle siguiente:

1) $1.000 \mathrm{~g} \mathrm{CR}-1113$

2) $1.000 \mathrm{~g} \mathrm{Setesa}-9$

3) $1.000 \mathrm{~g} \mathrm{CR}-5272$

4) 500 g CR-1113 y 500 g CR-5272
5) 500 g CR-1113 y 500 g Setesa-9

6) 500 g CR-5272 y 500 g Setesa-9

7) 333,3 g CR-1113/333,3 g CR-5272 y 333,3 g Setesa-9

\section{Diseño experimental}

Se utilizó un diseño de bloques completos al azar con seis repeticiones y dos réplicas por repetición.

Del lote de semilla certificada de cada variedad se prepararon seis sub-lotes de $5 \mathrm{~kg}$ cada uno, los que a su vez se dividieron en 10 porciones de $500 \mathrm{~g}$, para un total de 60. Cada una de estas porciones se unió con otra u otras, según lo determinaban los tratamientos, y su peso se ajustó a $1 \mathrm{~kg}$. La decisión de cuáles porciones unir se tomó aleatoriamente.

\section{Procedimiento de análisis}

Cada unidad experimental fue pasada por un determinador de impurezas "Carter Dockage Tester" y se registró su peso limpio (A en Figura 1). Posteriormente, se pasó una vez por un descascarador "McGill", el cual fue limpiado después de cada operación; los granos recuperados durante la limpieza se reintegraron a la unidad experimental correspondiente, la cual fue pesada (B en Figura 1) y luego procesada en un pulidor "McGill No.3" (30 seg con pesa de 0,91 kg (2 lb) y $15 \mathrm{seg}$ sin pesa en el brazo de presión, para blanqueado y pulido, respectivamente). El arroz pilado se colocó en un recipiente plástico; una vez a la temperatura ambiente, se pesó (C en Figura 1). El pulidor McGill fue limpiado después de cada operación y la semolina total producida fue reunida y pesada (D en Figura 1). Se utilizó una balanza de laboratorio con una resolución de 0,01 g.

De acuerdo con la norma vigente (MEIC 1998), arroz entero corresponde a todo grano de arroz pilado cuya longitud es igual o mayor a 3/4 de la longitud promedio de los granos sin quebraduras en la muestra. La cuantificación del porcentaje de entero, quebrado, quebrado grueso y puntilla se hizo sobre porciones de 100 g de arroz pilado, obtenidas con el divisor "Gamet", las cuales se clasificaron con el equipo "Rice Sizing Device"; para lo que se utilizaron las cribas 5, 6, 10 


\begin{tabular}{|c|c|c|c|c|c|c|}
\hline \multicolumn{7}{|c|}{ Unidad experimental limpia (A) } \\
\hline \multicolumn{6}{|c|}{ Arroz integral (B) } & \multirow[t]{5}{*}{ Cáscara } \\
\hline \multicolumn{3}{|c|}{ Arroz pilado $(\mathrm{C})$} & \multicolumn{3}{|c|}{ Semolina total (D) } & \\
\hline \multirow{3}{*}{$\begin{array}{c}\text { Entero } \\
\text { (1) }\end{array}$} & \multicolumn{2}{|c|}{ Quebrado } & \multirow{3}{*}{$\begin{array}{c}\text { Semolina } \\
\text { pura } \\
(4)\end{array}$} & \multirow{3}{*}{$\begin{array}{l}\text { Quebrado } \\
\text { muy fino } \\
\text { (5) }\end{array}$} & \multirow{3}{*}{$\begin{array}{c}\text { Cascarilla } \\
\text { molida } \\
(6)\end{array}$} & \\
\hline & Quebrado & Puntilla & & & & \\
\hline & grueso (2) & (3) & & & & \\
\hline
\end{tabular}

Figura 1. Esquema de la composición física del arroz en cáscara seco. Costa Rica, 2001.

y 12, siguiendo los procedimientos usuales. Las fracciones se revisaron visualmente para remover granos (enteros o quebrados) que no correspondieran a la fracción evaluada. Los pesos fueron determinados en una balanza con una resolución de $0,001 \mathrm{~g}$.

La semolina total de cada unidad experimental se colocó sobre una criba circular de laboratorio con aberturas de $0,99 \mathrm{~mm}$ de diámetro, con bandeja de fondo, la cual se agitó manualmente; se obtuvieron dos fracciones: una sobre la criba, conformada por pedazos pequeños de arroz quebrado (adelante se le denominará "quebrado muy fino") y restos de cascarilla molida; y en la bandeja de fondo, lo que se denomina en este estudio como "semolina pura".

El material sobre la criba se pasó por un soplador de laboratorio "Erickson modelo B" con una abertura de cuatro, para separar la cascarilla del quebrado muy fino. Además, se sometió a una inspección visual, a fin de remover cualquier contaminante no separado por el soplador. Los pesos fueron determinados en una balanza con una resolución de $0,01 \mathrm{~g}$.

\section{Variables de calidad molinera}

Las variables analizadas en este estudio se presentan en el Cuadro 1.

\section{Análisis de los datos}

Para analizar los tratamientos 4, 5, 6 y 7 y determinar si hubo o no efectos atribuibles al procesamiento de las mezclas, a cada una de las variables se les calculó el siguiente índice:

$$
I_{m}=\left(\frac{O}{E}-1\right) \times 100
$$

Donde:

$\mathrm{I}_{\mathrm{m}}=$ Índice del efecto del beneficiado de una mezcla sobre una variable.

$\mathrm{O}=$ el resultado obtenido para la mezcla.

$\mathrm{E}=$ el resultado esperado, que corresponde al promedio aritmético de los resultados de la variable en análisis, para cada una de las variedades en la mezcla (tratamientos 1, 2 ó 3).

El índice expresa este efecto de tres formas: índice igual a cero (sin efecto); índice positivo (resultado obtenido mayor que el esperado); e índice negativo (resultado obtenido menor que el esperado). Para las variables de rendimiento molinero, un índice positivo indica un efecto beneficioso del procesamiento de las variedades en mezcla; uno negativo, un efecto detrimental.

\section{RESULTADOS}

Al realizar el análisis estadístico de las variables de calidad molinera indicadas en el Cuadro 1 , no se detectaron diferencias significativas. Sin embargo, por ser ésta la primera vez que se estudia el efecto del beneficiado de mezclas de arroz, y además debido a que las diferencias observadas podrían ser de interés para la industria arrocera por su importancia económica, se decidió ahondar en su análisis. 
Cuadro 1. Variables de calidad molinera de arroz analizadas y su cálculo. Costa Rica. 2002.

\begin{tabular}{lrl}
\hline \multicolumn{1}{c}{ Variable } & Fórmula de cálculo 1 \\
\hline Rendimiento de Molino (RM) & $\mathrm{R}$ (M) $=\frac{1+2+3}{\mathrm{~A}} * 100$ \\
Rendimiento de arroz comercial (RAC) & $\mathrm{RAC}=\frac{1+2}{\mathrm{~A}} * 100$ \\
Índice de pilada (IP) & $\mathrm{IP}=\frac{1}{\mathrm{~A}} * 100$ \\
Porcentaje de semolina total (ST) & $\mathrm{ST}=\frac{\mathrm{D}}{\mathrm{B}} * 100$ \\
Porcentaje de semolina pura (SP) & $\mathrm{SP}=\frac{4}{\mathrm{~B}} * 100$ \\
Porcentaje de quebrado muy fino en la semolina (QMF) & $\mathrm{QW} \mathrm{F}=\frac{5}{\mathrm{~B}} * 100$ \\
Porcentaje de cascarilla en la semolina (CS) & $\mathrm{CS}=\frac{6}{\mathrm{~B}} * 100$ \\
Porcentaje de puntilla (P) & $\mathrm{Q}$ \\
\hline
\end{tabular}

${ }^{1}$ Los números y las letras en la parte derecha de las ecuaciones corresponden a los indicados en la Figura 1.

En el Cuadro 2 se presentan los valores observados y esperados de 10 variables evaluadas en este estudio.

En todas las figuras, las variables se presentan en tres grupos, separadas por líneas verticales; el primero, las variables relacionadas con la semolina (salvado) y sus componentes; el segundo, las variables relacionadas con el grano quebrado y sus componentes; y el tercero, las variables de rendimiento molinero. En general, los cambios observados en las variables de rendimiento molinero pueden ser explicados por los cambios en la producción de semolina y de grano quebrado.

\section{Mezcla de las tres variedades}

Como se observa en la Figura 2, el beneficiado de la mezcla de partes iguales de las tres variedades produjo un efecto detrimental en las variables de rendimiento molinero ( $\mathrm{I}_{\mathrm{m}}$ negativos), resultado de valores mayores a los esperados en todas las variables asociadas a los subproductos (semolina y quebrado).

En cuanto al Rendimiento de Molino, la disminución de $0,5 \mathrm{~g}$ de entero y quebrado por cada $100 \mathrm{~g}$ de arroz en cáscara en el valor observado es comparable 
Cuadro 2. Valores observados y esperados para 10 variables de calidad molinera en arroz. Costa Rica, 2002.

\begin{tabular}{|c|c|c|c|c|c|c|c|c|c|c|c|}
\hline \multicolumn{2}{|c|}{ Tratamiento } & \multirow{2}{*}{$\begin{array}{c}\text { Semoli- } \\
\text { na total } \\
(\%)\end{array}$} & \multirow{2}{*}{$\begin{array}{c}\begin{array}{c}\text { Semoli- } \\
\text { na pura } \\
(\%)\end{array} \\
6,33\end{array}$} & \multirow{2}{*}{$\begin{array}{c}\begin{array}{c}\text { Quebrado muy } \\
\text { fino en la } \\
\text { semolina (\%) }\end{array} \\
0,67\end{array}$} & \multirow{2}{*}{$\begin{array}{c}\begin{array}{c}\text { Cascarilla } \\
\text { en la semo- } \\
\text { lina }(\%)\end{array} \\
3,56\end{array}$} & \multirow{2}{*}{$\begin{array}{c}\text { Quebrado } \\
\text { (\%) }^{\mathbf{1}} \\
\\
19,43 \\
(13,88)\end{array}$} & \multirow{2}{*}{$\begin{array}{c}\begin{array}{c}\text { Quebrado } \\
\text { grueso } \\
(\%)^{\mathbf{1}}\end{array} \\
15,94 \\
(11,39)\end{array}$} & \multirow{2}{*}{$\begin{array}{c}\begin{array}{c}\text { Puntilla } \\
(\%)^{\mathbf{1}}\end{array} \\
3,48 \\
(2,49)\end{array}$} & \multirow{2}{*}{$\begin{array}{c}\begin{array}{c}\text { Ren- } \\
\text { dimiento } \\
\text { molino } \\
(\%)\end{array} \\
71,49\end{array}$} & \multirow{2}{*}{$\begin{array}{c}\begin{array}{c}\text { Rendimiento } \\
\text { de arroz } \\
\text { comercial } \\
(\%)\end{array} \\
69,01\end{array}$} & \multirow{2}{*}{$\begin{array}{c}\begin{array}{c}\text { Índice de } \\
\text { pilada } \\
(\%)\end{array} \\
57,62\end{array}$} \\
\hline CR-1113 & $\mathrm{O}$ & & & & & & & & & & \\
\hline Setesa-9 & $\mathrm{O}$ & 8,88 & 6,35 & 0,58 & 1,94 & $\begin{array}{c}19,10 \\
(14,60)\end{array}$ & $\begin{array}{c}16,53 \\
(11,59)\end{array}$ & $\begin{array}{c}2,57 \\
(3,01)\end{array}$ & 73,03 & 71,16 & 59,08 \\
\hline CR-5272 & $\mathrm{O}$ & 7,56 & 5,19 & 0,62 & 1,70 & $\begin{array}{c}19,49 \\
(13,95) \\
\end{array}$ & $\begin{array}{c}15,48 \\
(12,07)\end{array}$ & $\begin{array}{c}4,02 \\
(1,88) \\
\end{array}$ & 74,90 & 71,89 & 60,30 \\
\hline \multirow{2}{*}{$\begin{array}{l}\text { CR-1113 - } \\
\text { CR } 5272\end{array}$} & $\mathrm{O}$ & 9,19 & 5,73 & 0,69 & 2,71 & $\begin{array}{c}19,83 \\
(14,49)\end{array}$ & $\begin{array}{c}15,92 \\
(11,64)\end{array}$ & $\begin{array}{c}3,91 \\
(2,86)\end{array}$ & 73,12 & 70,26 & 58,63 \\
\hline & E & 9,07 & 5,76 & 0,64 & 2,63 & $\begin{array}{c}19,46 \\
(14,24)\end{array}$ & $\begin{array}{c}15,71 \\
(11,49)\end{array}$ & $\begin{array}{c}3,75 \\
(2,75) \\
\end{array}$ & 73,20 & 70,45 & 58,96 \\
\hline \multirow{2}{*}{$\begin{array}{l}\text { CR-1113 } \\
\text { - Setesa-9 }\end{array}$} & $\mathrm{O}$ & 9,68 & 6,16 & 0,63 & 2,85 & $\begin{array}{c}19,03 \\
(13,77)\end{array}$ & $\begin{array}{c}16,01 \\
(11,59)\end{array}$ & $\begin{array}{c}3,03 \\
(2,19)\end{array}$ & 72,39 & 70,20 & 58,62 \\
\hline & E & 9,73 & 6,34 & 0,62 & 2,75 & $\begin{array}{c}19,26 \\
(13,91)\end{array}$ & $\begin{array}{c}16,24 \\
(11,73)\end{array}$ & $\begin{array}{c}3,03 \\
(2,18)\end{array}$ & 72,26 & 70,08 & 58,35 \\
\hline \multirow{2}{*}{$\begin{array}{l}\text { CR-5272 } \\
\text { - Setesa-9 }\end{array}$} & $\mathrm{O}$ & 8,26 & 6,83 & 0,61 & 1,75 & $\begin{array}{c}19,34 \\
(14,30)\end{array}$ & $\begin{array}{c}15,97 \\
(11,80)\end{array}$ & $\begin{array}{c}3,38 \\
(2,49)\end{array}$ & 73,91 & 71,42 & 59,62 \\
\hline & E & 8,22 & 5,77 & 0,60 & 1,82 & $\begin{array}{c}19,30 \\
(14,27)\end{array}$ & $\begin{array}{c}16,00 \\
(11,83)\end{array}$ & $\begin{array}{c}3,29 \\
(2,44)\end{array}$ & 73,97 & 71,52 & 59,69 \\
\hline \multirow{2}{*}{$\begin{array}{l}\text { CR-1113 } \\
\text { - CR } 5272 \\
\text { - Setesa-9 }\end{array}$} & $\mathrm{O}$ & 9,09 & 5,97 & 0,66 & 2,44 & $\begin{array}{c}19,57 \\
(14,30)\end{array}$ & $\begin{array}{c}16,13 \\
(11,79)\end{array}$ & $\begin{array}{c}3,44 \\
(2,52)\end{array}$ & 73,10 & 70,59 & 58,80 \\
\hline & E & 9,60 & 5,95 & 0,62 & 2,40 & $\begin{array}{c}19,34 \\
(14,14)\end{array}$ & $\begin{array}{c}15,98 \\
(11,69)\end{array}$ & $\begin{array}{c}3,36 \\
(2,46)\end{array}$ & 73,15 & 70,68 & 59,00 \\
\hline
\end{tabular}

Nota: $\mathrm{O}=$ Observado y $\mathrm{E}=$ Esperado

${ }^{1}$ Los datos entre paréntesis se calcularon como porcentaje del peso de la unidad experimental después de haber sido limpiada.

a la suma de los pesos adicionales de semolina pura y quebrado muy fino por cada $100 \mathrm{~g}$ de arroz en cáscara (0,020 y 0,031 respectivamente).

Para esta mezcla, todas las variables del quebrado obtuvieron valores mayores que los esperados $\left(\mathrm{I}_{\mathrm{m}}>\right.$ $0)$, lo que provocó una disminución importante en el rendimiento de arroz comercial $\left(I_{m}=-0,14\right)$ y en el índice de pilada $\left(\mathrm{I}_{\mathrm{m}}=-0,34\right)$. Para el rendimiento de arroz comercial su disminución se puede explicar por la producción adicional de puntilla y de quebrado muy fino en la semolina. La disminución en el índice de pilada se puede explicar por la producción adicional de grano quebrado y de quebrado muy fino en la semolina. Las disminuciones detectadas en estas dos variables de rendimiento, expresadas en gramos, fueron explicadas en más de un $97 \%$ por el peso de las producciones adicionales de las fracciones que afectan el cálculo de cada variable de rendimiento (Figura 1 y Cuadro 1).

De lo anterior, se deriva que el efecto detrimental observado en las variables de rendimiento fue provocado por la producción de más fragmentos de arroz y, en mucho menor grado, por un pulido adicional. 


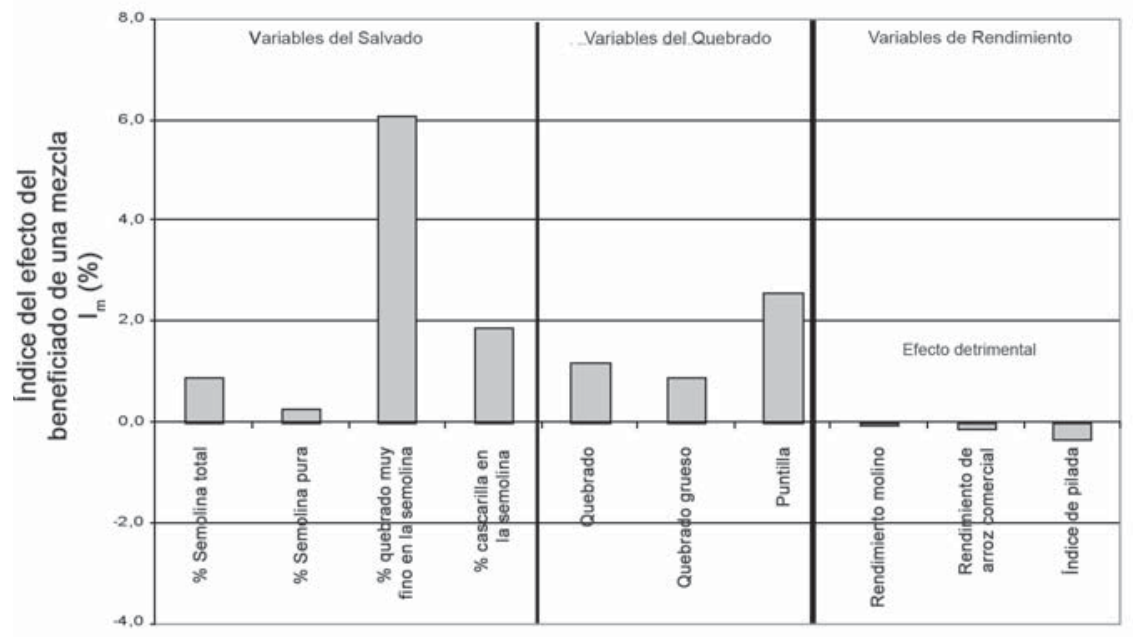

Figura 2. Desviaciones porcentuales entre los valores observados y los esperados $\left(I_{m}\right)$ de 10 variables de rendimiento molinero, cuando se benefició una mezcla de partes iguales de las variedades de arroz CR-1113, CR-5272 y Setesa 9. Costa Rica, 2002.

\section{Mezcla de CR-1113 y CR-5272}

$\mathrm{Al}$ igual que con la mezcla anterior, el beneficiado de partes iguales de CR-1113 y CR 5272 produjo un efecto detrimental en todas las variables de rendimiento molinero $\left(I_{m}=-0,10, I_{m}=-0,26, I_{m}=-0,56\right.$, para el rendimiento de molino (RM), el rendimiento de arroz comercial (RAC) y el índice de pilada (IP), respectivamente) (Figura 3).

Como se observa en esa figura, este efecto detrimental se explica por una producción adicional de quebrado muy fino en la semolina, de puntilla y de quebrado grueso. Por lo tanto, en esta mezcla el efecto

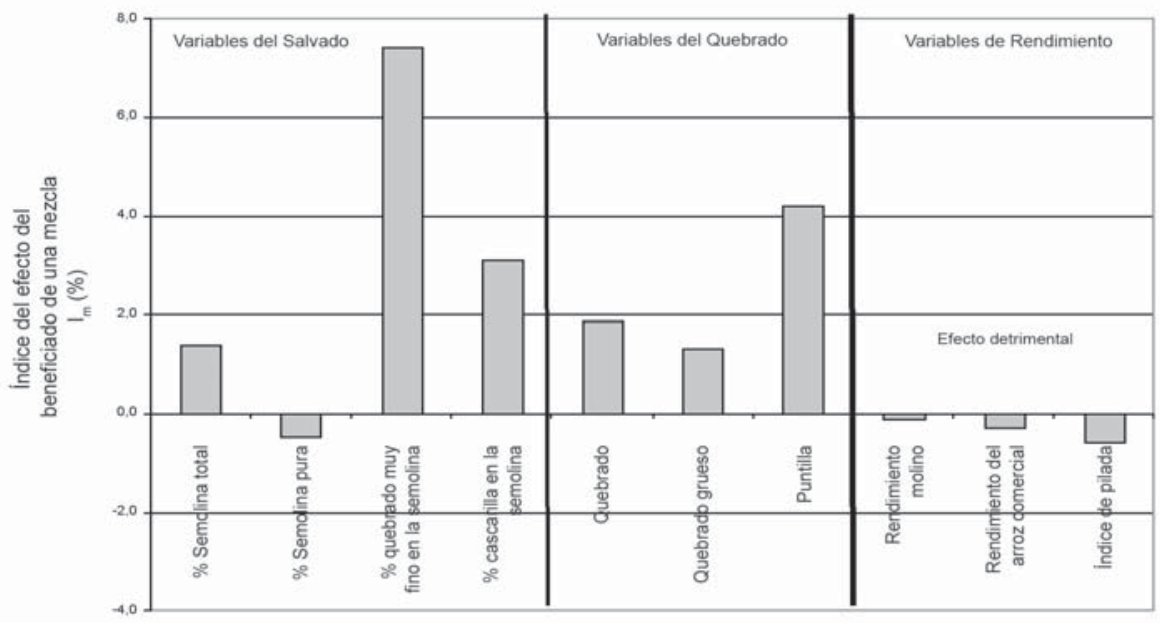

Figura 3. Desviaciones porcentuales entre los valores observados y los esperados $\left(I_{m}\right)$ para 10 variables en la mezcla de partes iguales de las variedades de arroz CR-1113 y CR5272. Costa Rica, 2002. 


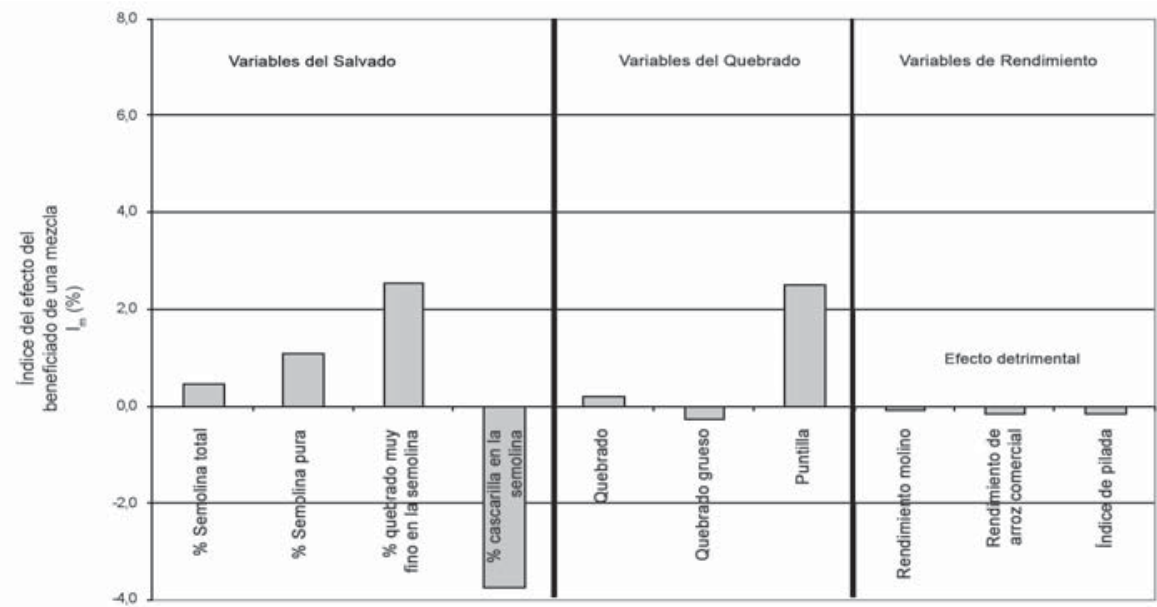

Figura 4. Desviaciones porcentuales entre los valores observados y los esperados (Im) para 10 variables en la mezcla de partes iguales de las variedades de arroz CR-5272 y Setesa 9. Costa Rica, 2002.

detrimental observado en las variables de rendimiento tuvo su origen en la producción de cantidades adicionales de fragmentos de arroz, efecto que no pudo ser contrarrestado por una producción menor que la esperada $\left(I_{m}=-0,48\right)$ de semolina pura.

\section{Mezcla de CR-5272 y SETESA-9}

Esta mezcla produjo rendimientos molineros ligeramente menores que los esperados $\left(\mathrm{I}_{\mathrm{m}}=-0,07 ; \mathrm{I}_{\mathrm{m}}=\right.$ - 0,15; $\mathrm{I}_{\mathrm{m}}=-0,13$, para RM, RAC e IP, respectivamente) (Figura 4). Esto se explica, por la tendencia de esta mezcla a producir cantidades adicionales de puntilla $\left(\mathrm{I}_{\mathrm{m}}=2,53\right)$; pero también por producciones adicionales de semolina pura $\left(\mathrm{I}_{\mathrm{m}}=1,09\right)$ y de quebrado muy fino en la semolina $\left(I_{m}=2,58\right)$; las que superaron en peso la menor producción de quebrado grueso $\left(\mathrm{I}_{\mathrm{m}}=-0,23\right)$.

De las tres mezclas que mostraron un efecto detrimental en las variables de rendimiento, ésta fue la que presentó un menor impacto. Al igual que en las otras mezclas con este tipo de efecto, esto se debió principalmente a la mayor producción de fragmentos de arroz, aunque en esta mezcla solo se produjeron cantidades adicionales de los fragmentos más pequeños (puntilla y quebrado muy fino).

\section{Mezcla de CR-1113 y SETESA-9}

El beneficiado de esta mezcla (Figura 5), al contrario de las anteriores, produjo un efecto beneficioso en todas las variables de rendimiento $\left(\mathrm{I}_{\mathrm{m}}\right.$ de 0,$17 ; 0,17$ y 0,46 para RM, RAC e IP, respectivamente). Esto se explica por una menor producción de quebrado grueso $\left(\mathrm{I}_{\mathrm{m}}=-1,41\right)$ y porque, contrariamente a las otras mezclas analizadas, no hubo producción adicional de puntilla $\left(\mathrm{I}_{\mathrm{m}}=0\right)$; porque la producción adicional de quebrado muy fino en la semolina fue la más pequeña $\left(\mathrm{I}_{\mathrm{m}}=1,18\right)$ y porque la producción de semolina pura presentó una importante reducción $\left(\mathrm{I}_{\mathrm{m}}=-2,78\right)$, posiblemente como consecuencia de un proceso incompleto de pulido por efecto de la mezcla. Una menor producción de subproductos explica valores más altos en las variables de rendimiento molinero.

La menor producción de semolina pura, transformada a una base igual a la de las variables de rendimiento, explica el aumento en el RM y el RAC. En 


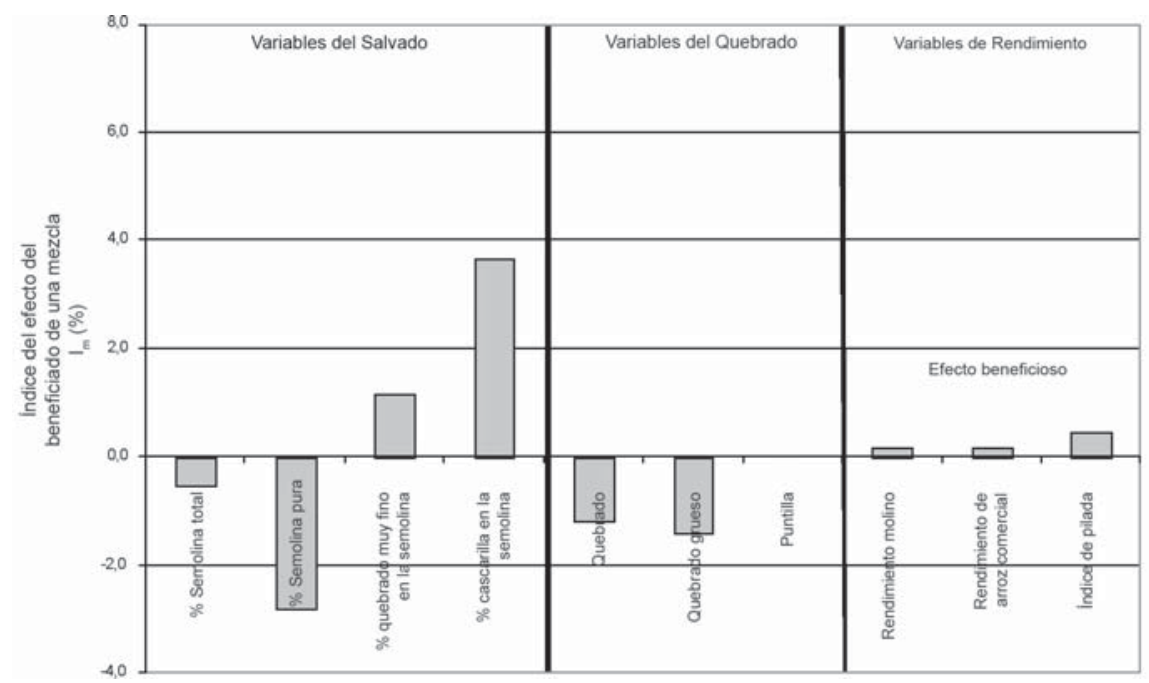

Figura 5. Desviaciones porcentuales entre los valores observados y los esperados $\left(\mathrm{I}_{\mathrm{m}}\right)$ para 10 variables en la mezcla de partes iguales de las variedades de arroz CR-1113 y Setesa 9. Costa Rica, 2002.

el caso del índice de pilada, la menor producción de semolina pura explica solo el 53\% de su incremento; el resto se explica, con la menor producción de quebrado grueso $\left(I_{m}=-1,41\right)$.

Esta menor producción de semolina pura sugiere que alguna de las dos variedades o ambas se pulen menos cuando se las procesa en mezcla; esto y la menor producción de grano quebrado apuntaron a que estas dos variedades presentan características que las hacen compatibles para el beneficiado en mezcla.

\section{CR-5272}

Esta variedad fue la que presentó la menor proporción de capas externas removidas durante el blanqueado y pulido, a saber, 4,22 \% de semolina pura, y los valores más altos para las variables de rendimiento molinero (Cuadro 2).

En todas las mezclas en que participó CR-5272 se obtuvieron valores del $\mathrm{I}_{\mathrm{m}}$ negativos para todas las variables de rendimiento molinero, lo que se asoció con producciones consistentemente mayores a las esperadas de puntilla y de quebrado muy fino.

\section{CR-1113}

La única tendencia consistente que presentó esta variedad cuando se benefició en mezcla con otras, fue que se produjeron $I_{m}$ positivos para el quebrado muy fino y para la cascarilla en la semolina (Figuras 2, 3 y 5). No obstante, lo más relevante de esta variedad es que integró la única mezcla (con Setesa 9) considerada beneficiosa, pero también participó de la mezcla más perjudicial (con CR-5272).

\section{Setesa-9}

Esta variedad formó parte de la única mezcla que mejoró los rendimientos molineros, a pesar de que, produjo mayor cantidad de quebrado muy fino que el esperado en todas las mezclas en que participó. Cuando las mezclas fueron de solo dos variedades, produjo consistentemente menos quebrado grueso. 


\section{Diferencias en las dimensiones de los granos}

La OFIARROZ (1994, datos sin publicar) realizó un estudio de la relación largo/ancho de las principales variedades de arroz cultivadas en Costa Rica. Con esa información se confeccionó el Cuadro 3, que resume las diferencias de interés para las variedades utilizadas en este trabajo.

Cuadro 3. Diferencias en el largo y ancho promedio de los granos de arroz integral de las variedades de arroz CR-1113, CR-5272 y Setesa 91. Costa Rica, 2002.

\begin{tabular}{ccc}
\hline $\begin{array}{c}\text { Variedades } \\
\text { en mezcla }\end{array}$ & $\begin{array}{c}\text { Diferencias en } \\
\operatorname{largo}^{2}\end{array}$ & $\begin{array}{c}\text { Diferencias en } \\
\text { ancho }^{2}\end{array}$ \\
\hline
\end{tabular}

CR-1113 - Setesa 9 2,4 \% mayor CR-1113 2,3\% mayor CR-1113 CR-5272 - Setesa $9 \quad 0,0 \%$ (iguales) $\quad 7,4 \%$ mayor Setesa 9 CR-1113 - CR-5272 2,4\% mayor CR-1113 9,9\% mayor CR-1113

${ }^{1}$ Dimensiones en milímetros.

2 Dimensión mayor - Dimensión menor / Dimensión menor x 100.

\section{DISCUSIÓN}

En todas las mezclas evaluadas se observó una clara tendencia a producir más quebrado muy fino en la semolina que el esperado (Figuras 2, 3, 4 y 5); y en tres de las cuatro mezclas se produjo más puntilla que la esperada (Figuras 2, 3 y 4). Por el contrario, en dos mezclas se produjo menos grano quebrado grueso y en una los rendimientos molineros mejoraron.

Estos y otros resultados de este experimento demuestran que las variables de rendimiento molinero son afectadas de manera diversa cuando se benefician mezclas de variedades. En las mezclas perjudiciales, predominó como explicación de los rendimientos menores el que se produjeran cantidades adicionales de puntilla y de quebrado muy fino; también en dos de estas mezclas se produjo más quebrado grueso y más semolina pura.

Estos resultados sugieren que las interacciones entre los granos de dos o más variedades durante el blanqueado y pulido del arroz provocan que un porcentaje pequeño de éstos se quiebren. Una explicación de ésto puede encontrarse en lo que informan Sun y
Siebenmorgen (1993), en el sentido de que el quebrado fue alto en las fracciones de granos más delgados de un mismo lote de arroz. Al mezclar en el presente experimento granos de dos o más variedades, las diferencias en el ancho de los granos de las mezclas definitivamente aumentaron (Cuadro 3), por lo que podría haberse dado en este experimento que los granos más delgados en las mezclas hayan sido los que se quebraron en una mayor proporción.

Las diferencias en el ancho promedio de las variedades evaluadas en este experimento sugieren que pequeñas diferencias como la que presenta la mezcla CR-1113-Setesa-9 no son perjudiciales y aún podrían ser beneficiosas. Pero, que a partir de cierto punto, estas diferencias resultan perjudiciales. Las diferencias en el largo de los granos no parecen tener relación con los cambios en las variables de rendimiento.

Las consecuencias del efecto detrimental causado por el procesamiento de la mezcla CR 1113-CR-5272, la cual se clasificó como la más dañina, se estimó en una disminución del rendimiento de arroz comercial de 0,14 kg por saco de 73,6 kg de arroz en granza seca y limpia (unidad de medida para arroz en cáscara utilizada en Costa Rica). Dado que el precio de industrial a mayorista de arroz 80/20 era en el 2002 de \$ 0,46 por kilo, para un silo de 1.472 t (20.000 sacos de 73,6 kg) la pérdida fue calculada en \$ 1300 por causa de este fenómeno, o sea, aproximadamente $\$ 1$ por tonelada métrica. Esta pérdida es importante que la eviten los molinos arroceros, para lo que pueden tomar en cuenta los resultados de este experimento.

\section{CONCLUSIONES}

El procesamiento de mezclas de las tres variedades de arroz más importantes en Costa Rica durante la consecha 1999-2000, demostró que se pueden producir tanto efectos perjudiciales como beneficiosos en las principales variables de calidad molinera.

Todas las mezclas evaluadas (cuatro) presentaron un incremento en la cantidad de quebrado muy fino en la semolina; en tres de éstas se produjo más puntilla, y en dos, más quebrado grueso. En las mezclas en las que participó CR5272, estos efectos tuvieron una mayor magnitud. 


\section{RECOMENDACIONES}

Conviene que se considere el efecto del beneficiado de mezclas de variedades de arroz, y se estudien otras proporciones y otras variedades, para así tomar acciones que minimicen los efectos detrimentales y se maximicen los beneficiosos. Podría entonces, resultar conveniente que los molinos arroceros valoren la conveniencia del almacenamiento y procesamiento por separado de alguna variedad, como sería el caso de CR 5272, ya que, como se observó en este trabajo, podría disminuir el potencial productivo de las mezclas.

También es importante que en futuras investigaciones se tomen en cuenta variables como elasticidad y dureza de los granos, lo que permitirá dar una mejor explicación a los fenómenos encontrados. De igual manera, debería utilizarse un equipo para determinar o estimar el grado de pulido del arroz, para ajustar los tiempos de blanqueado y pulido a las necesidades de las mezclas a evaluar, así como considerar la relación de las diferencias en el ancho y en el largo de las variedades en mezcla con los efectos observados en este experimento.

\section{LITERATURA CITADA}

ARRIETA, J. 1994. Metodología para estimar la extracción de las capas externas del arroz integral durante la etapa de beneficiado. Tesis Escuela de Fitotecnia. Facultad de Agronomía. San José, Costa Rica. Universidad de Costa Rica. p. 45-58.

CASTILLO, A.; GAVIRIA, J. 2000. Molinería de arroz en los trópicos. Estudios y Diseños Agroindustriales Ltda. (Ediagro). Bogotá, Colombia. p. 167.

CRUZ, M. 2002. Efecto del beneficiado de mezclas de las variedades de arroz CR-1113, CR-5272 y Setesa-9 sobre variables de rendimiento molinero. Tesis Escuela de Agronomía. Facultad de Ciencias Agroalimentarias. San José, Costa Rica, Universidad de Costa Rica. 71 p.

MEIC (Ministerio de Economía Industria y Comercio, CR). 1998. RTCR 201:1998. Arroz en cáscara. Especificaciones y métodos de análisis. San José, Costa Rica. 13 p.

OFIARROZ (Oficina del Arroz, CR). 1994. Caracterización del largo y del ancho promedio de los granos de arroz integral de las variedades CR-1113, CR-5272 y Setesa 9. San José, Costa Rica. Datos sin publicar. $59 \mathrm{p}$.

2000. Informe anual. San José, Costa Rica.

SUN, H.; SIEBENMORGEN, T. J. 1993. Milling characteristics of various rough rice kernel thickness fractions. American Association of Cereal Chemists 70(6):727-733.

ZELEDÓN, M.; CORRALES, V. 1997. Efecto del tiempo de blanqueado del arroz en la sobrevivencia de Rhyzopertha dominica, en la remoción de las capas externas y en el porcentaje de grano quebrado. Agronomía Costarricense 21(2):171-178. 